\title{
A Comparative Study to Visualize PtdIns(4,5) P2 and PtdIns(3,4,5) P3 in MDA-MB-231 Breast Cancer Cell Line
}

\author{
Dhurgham Al-Fahad*1, Bandar Al-Harbi², Yahya Abbas ${ }^{3,}$ Firas Al-Yaseen ${ }^{4}$
}

\begin{abstract}
Background: Phosphatidylinositol 3,4,5-trisphosphate [PtdIns(3,4,5) P3) and Phosphatidylinositol 4,5trisphosphate (PtdIns(4,5) P2] form an insignificant amount of phospholipids but play important roles in controlling membrane-bound signalling. Little attention has been given to visualize and monitor changes or differences in the local generation of PtdIns(4,5) P2 and PtdIns(3,4,5) P3 in the cell membranes of MDAMB-231 breast cancer cell lines.

Methods: PLC81-PH-GFP and Btk-PH-GFP were used as biosensors to detected PtdIns(4,5) P2 and PtdIns(3,4,5)P3 respectively. These biosensors and antibodies were transfected, immuostained and then visualized by confocal microscopy on different cell surfaces.

Results: Our results showed that PLC $81-\mathrm{PH}-\mathrm{GFP} / \mathrm{mCherry}$ was localized at the cell membrane, while BtkPH-GFP/mCherry was sometimes localized at the cell membrane but there was also a large amount of fluorescence present in the cytosol and nucleus. Our results also showed that the cells that expressed low levels of Btk-PH-GFP the fluorescence was predominantly localised to the cell membrane. While the cells that expressed high levels of Btk-PH-GFP the fluorescence was localization in the cytosol and cell membrane. Our results demonstrated that both anti-PtdIns(4,5)P2 and anti-PtdIns(3,4,5)P3 antibodies were localized everywhere in cell.
\end{abstract}

Conclusions: Our results suggest that PLC $\delta 1-\mathrm{PH}-\mathrm{GFP}$ and Btk-PH-GFP/mCherry have more specificity, reliability, suitability and accuracy than antibodies in binding with and detecting PtdIns(4,5)P2 and PtdIns(3,4,5)P3 and in studying the molecular dynamics of phospholipids in live and fixed cells.

Keywords: Antibodies, Biosensors, MDA-MB-231, Phosphatidylinositol.

\section{Introduction}

Phospholipids are the main constituent of all cell membranes and form lipid bilayers due to their amphiphilic nature. The phospholipid molecule contains two hydrophobic fatty acid "tails" and a hydrophilic "head" containing a phosphate group. The two constituents are combined together with a glycerol molecule. The phosphate groups can be amended with simple organic molecules such as choline or inositol (1).

Pleckstrin Homology (PH) domains consist of around 120 amino acids and have a seven- strand $\beta$-barrel which forms two anti-parallel $\beta$ sheets and a $C$-terminal $\alpha$ helix. The $\beta 1, \beta 2$, $\beta 3$ and $\beta 4$ loops form specific sites to bind with the inositol ring of phosphoinositide (2). Approximately $10 \%$ of all $\mathrm{PH}$ domains have high affinity and specificity to bind with phosphoinositide (3). PtdIns(4,5) P2 has high affinity and specificity to bind with phospholipase $\mathrm{C}$ delta (PLC $\delta$ ) $\mathrm{PH}$ domain and PtdIns(3,4,5) P3 also has high affinity and specificity to bind with Bruton's tyrosine kinase (Btk) PH domain due to neighbouring 
phosphates in their inositol ring (4). However, other types of phosphoinositide, such as PtdIns3P, PtdIns5P and PtdIns(3,5)P2 bind with another type of domains, such as FYVE domains, PHD finger and PX (3). This diversity in the specificity of phosphoinositide to associate with different types of $\mathrm{PH}$ domain containing proteins is important due to the recruitment of a variety of proteins to the cell membrane to perform their function and interact with other signalling pathways (3). Some of these proteins phosphorylate the inositol ring to generate a new phosphoinositide or produce new second messengers, such PI3K phosphorylate PtdIns(4,5)P2 to PtdIns(3,4,5)P3 and PLC cleaves PtdIns(4,5)P2 to Inositol trisphosphate (IP3) and diacylglycerol (DAG). Other proteins dephosphorylate sites on the inositol ring, such as PTEN dephosphorylates PtdIns(3,4,5)P3 to PtdIns(4,5)P2 (2,3). Phospholipid signalling plays a key role in the regulation of a substantial number of cellular processes, such as migration, cell proliferation, apoptosis, metabolism, and focal adhesions $(5,6,7)$.

Phosphatidylinositols (PtdIns) are a type of phospholipids that exist in the cytoplasmic layer of cell (8). They consist of a glycerol group which is esterified to two fatty acid chains and a phosphate group that binds to a polar inositol head group and extends into the cytoplasm. Phosphorylated forms of Phosphatidylinositols are phosphoinositides and constitute a small percentage of the lipid in the plasma membrane. The amount of PtdIns has been evaluated and found that PI forms $\sim 10-20 \% \quad(\mathrm{~mol} \%)$ of total cellular phospholipids, (PtdIns(4,5) P2) form $\sim 0.2-$ $1 \%$, and PtdIns(3,4,5) P3 constitutes approximately $2-5 \%$ of the PtdIns(4,5) P2 (8). Until recently the direct detection of (PtdIns(4,5) P2) and PtdIns(3,4,5) P3 at cell membrane has been difficult. Therefore, in this study we aimed to use biosensors and antibodies to visualize them and examine which one is better to use.

\section{Materials and Methods \\ Cell lines and cell culture}

Experiments were performed using MDAMB-231(ATCC $®$ HTB-26 ${ }^{\mathrm{TM}}$ ), and MCF7 (ATCC $®$ HTB-22 ${ }^{\mathrm{TM}}$ ) human adenocarcinoma cells were obtained from the American Type Culture Collection (ATCC, Manassas, VA). MDA-MB-231 cells were tested by Mycoplasma kit (Cat No. K1-0210, EZ-PCR Mycoplasma Test Kit, 20 assays, Project. A3088606MDA-MB-231 cells were transferred into $10 \mathrm{ml}$ of DMEM (Gibco). The flask was incubated at $37{ }^{\circ} \mathrm{C}, 5 \% \mathrm{CO}_{2}$ and $95 \%$ humidity until the cells become confluent. The MDA-MB-231 cells were grown in T75 flasks and cell passaging was used to maintain growth of cells. To count cells, a haemocytometer was used to determine the number of cells for each experiment and a formula was used:

No. of Cells $/ \mathrm{ml}=$ (average No. of cells counted/No. of cells wanted) $\times 10^{4} \mathrm{x}$ dilution factor.

\section{Transfection}

The ibidi dish was coated with one $\mathrm{ml}$ of 10 $\mu \mathrm{g} / \mathrm{ml} \mathrm{v} / \mathrm{v}$ collagen (BD Bioscience), then seeded with $1 \times 10^{5}$ MDA-MB-231 cells for 24 hours at $70 \%$ confluence before transfection. Next day, $3 \mu \mathrm{g}$ of PLC $\delta 1-P H-G F P$ (Addgene; Plasmid \#21179) or Btk-PH- GFP (Addgene; Plasmid \#51463) was added in $100 \mu \mathrm{l}$ of serum-free media in labelled Eppendorf tubes. $6 \mu$ l Polyethyleneimine (PEI) (Sigma Aldrich) transfection reagent was added to each labelled tube and then immediately mixed by vortex for 10 seconds. The labelled tubes were incubated for 15 minutes in the culture hood. The Plasmid/PEI mixture was added into each well which contains $2 \mathrm{ml}$ of complete media. The plate was gently swirled and incubated overnight.

\section{Confocal microscopy and live cell imaging}

The ibidi dish was put in the confocal chamber and the chamber was connected to $\mathrm{CO}_{2}$ supply needle and warmed at $37^{\circ} \mathrm{C}$, one hour prior to use. The $488 \mathrm{~nm}$ and $568 \mathrm{~nm}$ channels of 
confocal microscopy were used for GFP-C1PLCdelta-PHand Btk-PH-GFP or mCherry. Laser intensity and exposure time were adjusted to get rid of noise background.

\section{Immunostaining}

In order to visualize PtdIns(4,5)P2(Echelon Biosciences; Z-P045) or PtdIns(3,4,5)P3 (Echelon Biosciences; Z-P345), it is important to coat the coverslip with $1 \mathrm{ml}$ of $10 \mu \mathrm{g} / \mathrm{ml} \mathrm{v} / \mathrm{v}$ fibronectin(BD Bioscience). The cells were fixed by one $\mathrm{ml}$ of 4\% PFA (Sigma Aldrich) for 20 minutes at room temperature. The cells were incubated with Anti- PtdIns(4,5) P2 and Anti- PtdIns(3,4,5) P3 antibodies (1:10, $2 \mu 1$ antibody, $200 \mu \mathrm{l}$ PBS (Phosphate-buffered saline), $4 \mu 12 \%$ goat serum) for each well around one hour. The cells were washed three times with PBS for 10 minutes and then stained with 546 Alexa Fluor anti-Rabbit solution (1:10, $2 \mu 1$ antibody, $200 \mu \mathrm{l}$ PBS, $4 \mu 12 \%$ goat serum) for one hour in the dark. The cells were
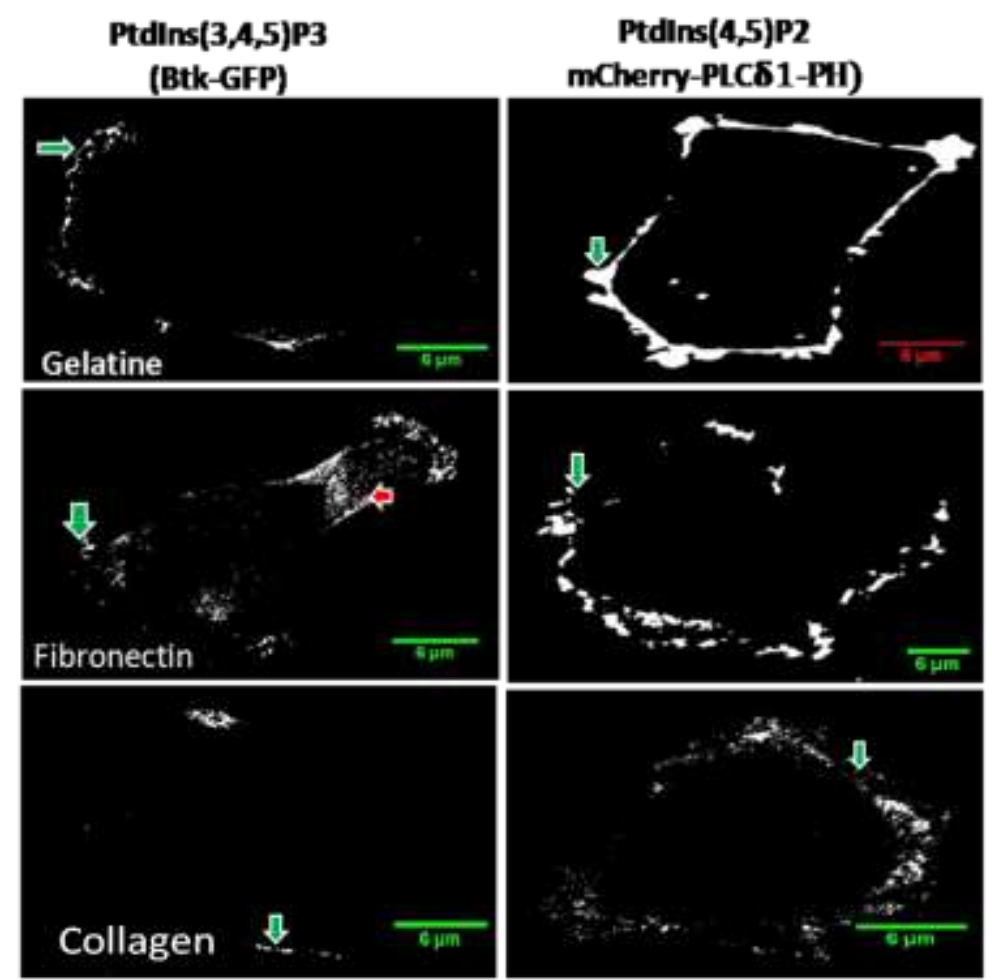

Fig. 1. visualisation of PtdIns(4,5)P2 and PtdIns(3,4,5)P3on the variety of surfaces in fixed cells: Confocal images of MDA-MB231 cells expressing the PLC $81-G F P$ or mCherry and Btk-PH-GFP were used as biosensors to identify PtdIns(4,5)P2 and PtdIns(3,4,5)P3lipids in the plasma membrane of MDA-MB-321 cells respectively. MDA-MB-321 cells were seeded on the variety of surfaces (gelatine $0.2 \%$, fibronectin $20 \mu \mathrm{g} / \mathrm{ml}$ and collagen $2 \mathrm{mg} / \mathrm{ml}$ ). Then, cells were transfected with PLC $\delta 1-G F P$ or mCherry and Btk-PH-GFP or mCherry after stimulating PtdIns(3,4,5) P3productionwith EGF (100 ng/ml) for 5 minutes. Then, cells were fixed with paraformaldehyde to visualise PtdIns(4,5) P2 and PtdIns(3,4,5) P3. The green arrow indicates the localisation of PtdIns(4,5)P2 and PtdIns(3,4,5)P3 on the cell membrane and the red arrow indicates the localisation of PtdIns(3,4,5)P3in the cytosol. Three independent experiments were performed, representative pictures are shown. 


\section{Effect of Btk-PH-GFP overexpression}

MDA-MB-231 cells were seeded and transfected. Our results also showed that the cells that expressed low levels of Btk-PH-GFP the fluorescence was predominantly localized to the cell membrane (Fig. 2). While the cells that expressed high levels of Btk-PH-GFP there was localization in the cytosol and cell membrane (Fig. 2). The fluorescence intensity in the cell membrane and cytosol were measured to compare the fluorescence intensity between these. In cells that expressed low levels of Btk- PH-GFP, the peak of
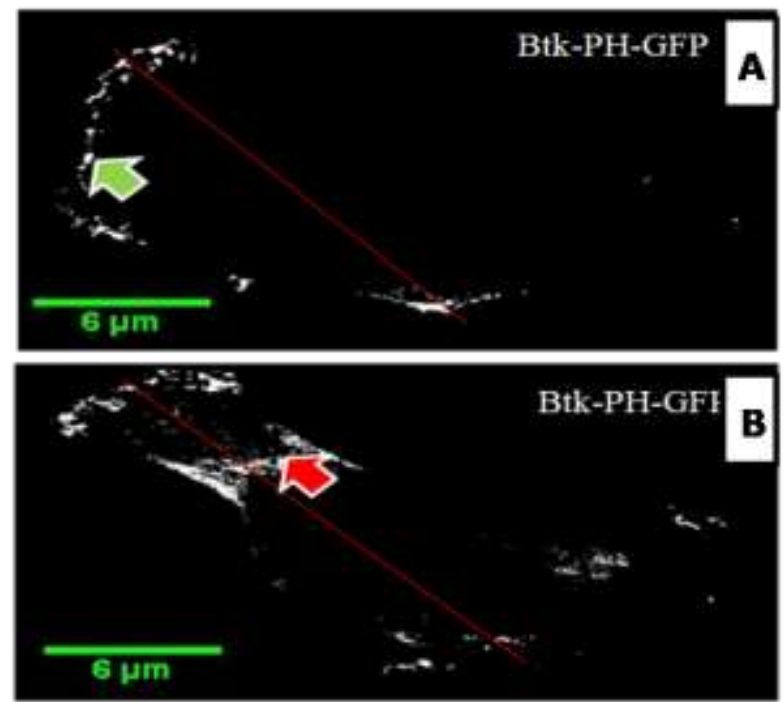

fluorescence intensity in the cell membrane was around 150 levels of pixel intensity, while in the cytosol it was around 10 levels of pixel intensity. This referred that localization of BtkPH-GFP in cell membrane was higher than in the cytosol (Fig. 2). In cells that expressed high levels of Btk-PH-GFP, the peak of intensity in the cell membrane was around 100 levels of pixel intensity, while in the cytosol it was around 125 levels of pixel intensity. This also referred that localization of Btk-PH-GFP in the cytosol was higher than in the cell membrane (Fig. 2).
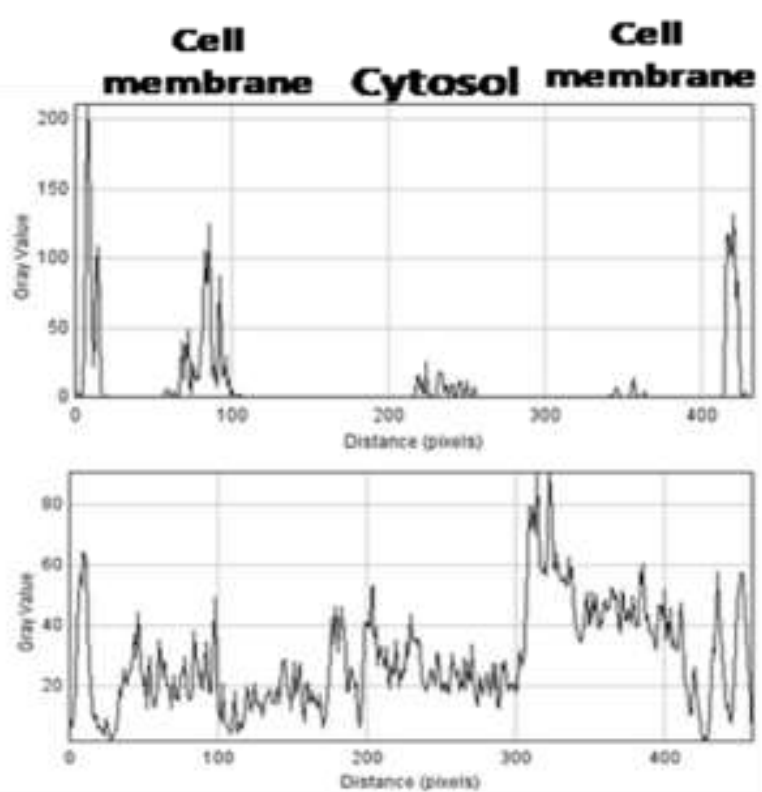

Fig. 2. Effect of Btk-PH-GFP overexpression in the cell. Confocal images of MDA-MB-231 cells that were transfected with BtkPH-GFP for 24-hour, A) At low expression levels, the peak of intensity in the cell membrane was higher than in the cytosol. B) At high expression levels, the peak of intensity in the cell membrane and cytosol was similar. The white arrow indicates the localisation of PtdIns (3,4,5) P3in the cell membrane and the red arrow indicates the localisation of PtdIns $(3,4,5)$ P3in the cytosol. The red line across cell refers to the measurement of intensity in the cell membrane and cytosol of the cell. Data are a representative of three independent experiments in which 10 cells were measured.

\section{Comparison between antibodies and $\mathrm{PH}$ - domains to detect PtdIns $(3,4,5)$ P3and PtdIns (4,5)P2}

MDA-MB-231 cells were seeded on fibronectin $20 \mu \mathrm{g} / \mathrm{ml}$, then fixed with paraformaldehyde and stained with anti- PtdIns(4,5) P2 and antiPtdIns(3,4,5) P3 antibodies. The results showed that both anti-PtdIns(4,5) P2 and antiPtdIns(3,4,5) P3 antibodies were localized in the cytosol and cell membrane (Fig. 3). The fluorescence intensity in the cell membrane and cytosol of cell were measured to compare between these. The data showed that the peak of fluorescence intensity of PLC 81 -PH-GFP in the cell membrane was around 300 levels of pixel intensity and in the cytosol, it was 0 levels of pixel intensity (Fig. 3). While the peak of fluorescence intensity of anti- PtdIns(4,5) P2 antibody in both cell membrane and cytosol was around 250 levels of pixel intensity (Fig. 3). This referred that PLC 1 1-PH-GFP had more specificity to bind with and detect PtdIns $(4,5)$ $\mathrm{P} 2$. The peak of intensity of Btk-PH-GFP in the cell membrane was around 170 levels of pixel intensity and in the cytosol, it was around 20 levels of pixel intensity (Fig. 4). While the peak 
of intensity of anti- PtdIns(3,4,5) P3 antibody in both cell membrane and cytosol was around 60 levels of pixel intensity (Fig. 4). This also

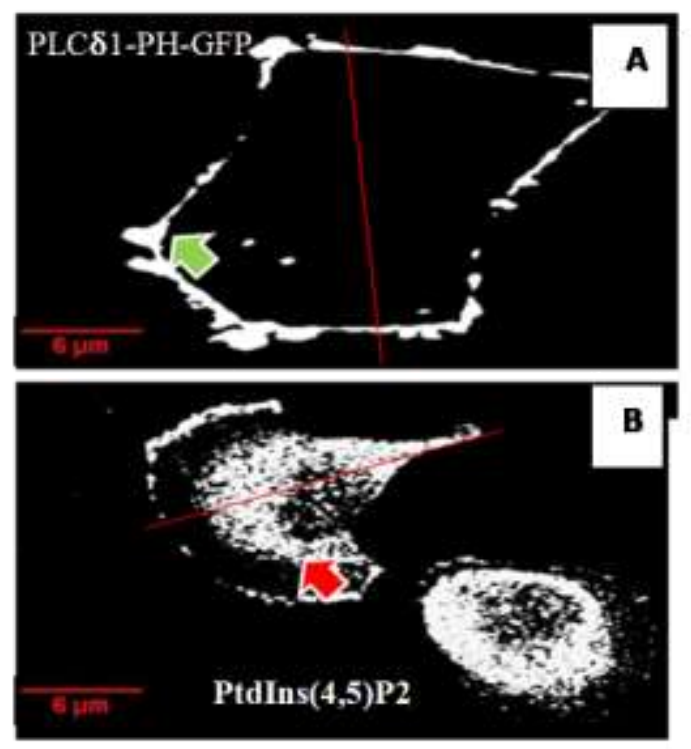

referred that Btk-PH-GFP had more specificity to bind with and detect $\operatorname{PtdIns}(3,4,5) \mathrm{P} 3$.
Cell Cell membrane Cytosol membrane
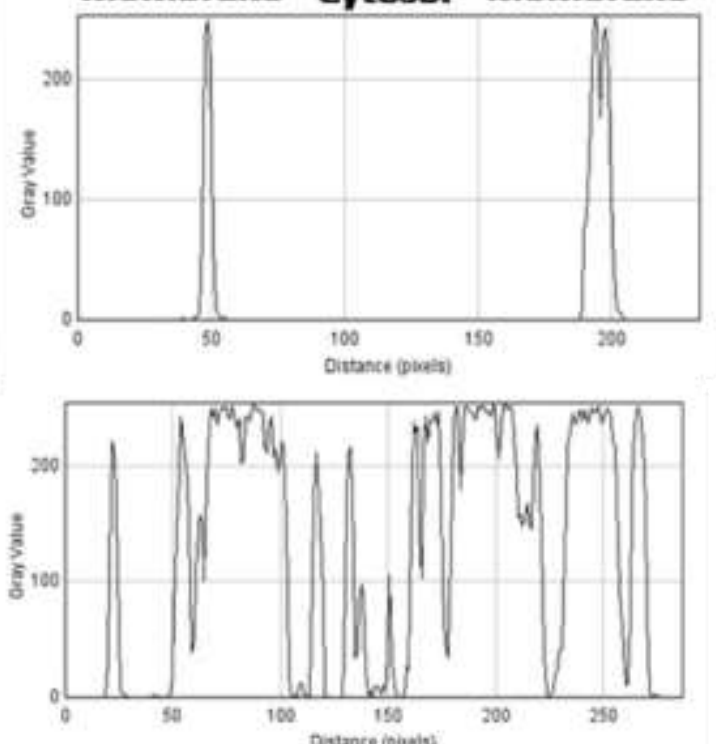

Fig. 3. Quantification of PtdIns(4,5) P2 localisation. A) MDA-MB-231 cells were transfected with PLC $81-\mathrm{PH}-\mathrm{GFP}$ and fixed with paraformaldehyde. Quantification of intensity in the cell membrane and cytosol of the cell. The peak of intensity was only in the cell membrane. B) MDA-MB-231 cells were stained with anti-PtdIns(4,5) P2antibody and fixed with paraformaldehyde. Quantification of intensity in the cell membrane and cytosol of the cell. The peak of intensity in the cell membrane and cytosol was similar. Data are representative of three independent experiments in which 10 cells were measured.
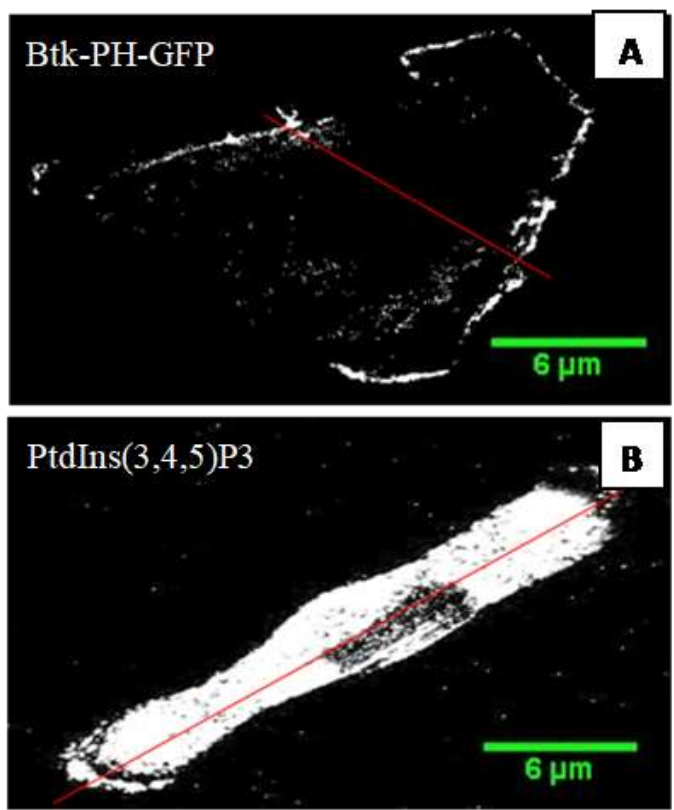

Cell

Cell membrane Cytosol membrane

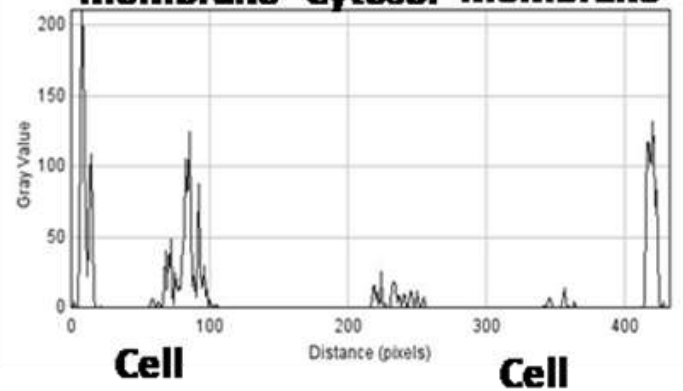

membrane Cytosol membrane

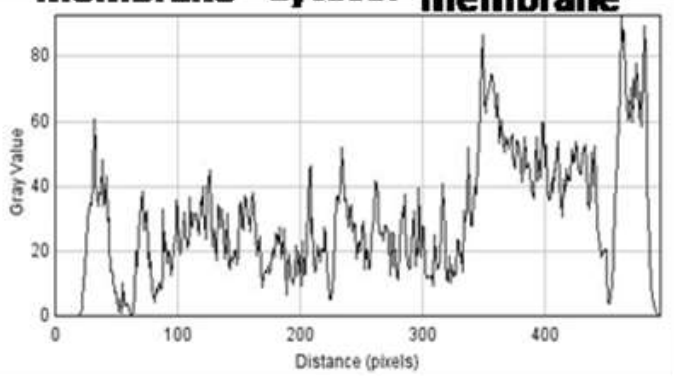

Fig. 4. Quantification of PtdIns(3,4,5)P3 localization. A) MDA-MB-231 cells were transfected with Btk-PH-GFP and fixed with paraformaldehyde. Quantification of the intensity in the cell membrane and cytosol. The peak of intensity in the cell membrane was higher than in the cytosol. B) MDA-MB-231 cells were stained with anti-PtdIns(3,4,5) P3antibody and then fixed with paraformaldehyde. Quantification of the intensity in the cell membrane and cytosol of the cell. The peak of intensity in the cell membrane and cytosol was similar. Data are representative of three independent experiments in which 10 cells were measured. 


\section{Discussion}

The aim of this study was to visualize and monitor the changes in levels of $\operatorname{PtdIns}(4,5) \mathrm{P} 2$ and PtdIns(3,4,5)P3 directly in MDA-MB-231 cells. Our data showed that PLC 1 1-PH-GFP which binds PtdIns(4,5)P2 (Fig. 1) was almost entirely recruited to the cell membrane in both the fixed and the live cells (Fig. 1), while an anti-PtdIns(4,5)P2antibody was localized throughout the cells, not just in the membrane (Fig. 2B). The probe Btk-PH-GFP for PtdIns $(3,4,5)$ P3 was sometimes localized at the cell membrane, but in other cells there was a large amount of fluorescence present in the cytosol and nucleus in both the fixed and the live cells. The anti-PtdIns(3,4,5) P3 antibody was localized throughout the cell.

Many biosensors have been designed to detect PtdIns(3,4,5)P3, such as the cytosolic regulator of adenyl cyclase (CRAC) protein $(8,9)$ and Akt-PH domain. Although Akt-PH domain is considered to be a good reporter, it also binds with PtdIns(4,5) P2 (10). Btk-PH domain is considered to be the best biosensor to visualize PtdIns(3,4,5)P3 as it has more affinity with, and specificity for PtdIns(3,4,5)P3 (1113). Our results also showed in transfected cells that expressed only a small amount of Btk-PHGFP, the majority of the GFP was recruited to cell membrane. MDA-MB-231 cells were transfected with Btk-PH-GFP for 24 hours, then the fluorescence intensity was measured in both transfected cells that expressed a small amount and more of Btk-PH-GFP.

Our results showed that transfected cells that expressed a small amount of the peak of fluorescence intensity in the cell membrane was higher than cytosol in the live and the fixed cells. While transfected cells that expressed more Btk-PH-GFP showed localization of the probe throughout the cell, and the peak of fluorescence intensity in the cell membrane and cytosol was slightly different in the live and the fixed cells. This may mean that Btk-PH-GFP interacts with PtdIns $(3,4,5) \mathrm{P} 3$ but it depends on the availability of PtdIns(3,4,5) P3 in the cell membrane to recruit Btk-PH-GFP to the cell membrane. Therefore, in transfected cells that expressed more Btk-PH, it is likely to saturate the available amount of PtdIns(3,4,5) P3 pools in the cell membrane, and the rest either binds with other types of inositides or remains in the cytoplasm (13).

There are many possible reasons why PLC $81-\mathrm{PH}$ and Btk-PH were recruited to the cell membrane differently. First, the abundance of PtdIns(3,4,5) P3 is much less than PtdIns(4,5) P2 in the cell membrane (14). PI $(, 4,5) \quad$ P2 forms around 5,000-20,000 molecules $/ \mu \mathrm{m}^{2}$, while PtdIns(3,4,5) P3forms only $2-5 \%$ of PtdIns $(4,5)$ P2 $(8,15)$. Therefore, our results showed that PLC $\delta 1-\mathrm{PH}-\mathrm{GFP}$ was almost entirely recruited to the cell membrane in both fixed and live cells due to an enrichment of PtdIns(4,5) P2in the cell membrane. Second, it has been supposed that there is the compensatory increase in the $\operatorname{PtdIns}(4,5) \mathrm{P} 2$ amount in the cells expressing large amount of proteins. This compensatory increase occurs rarely with $\operatorname{PtdIns}(3,4,5) \mathrm{P} 3$ due to its dependence on the certain stimuli, including PtdIns(3,4,5)P3 synthesis mainly relies on the presence of PI3K activity. Therefore, whenever $\mathrm{PI} 3 \mathrm{Ks}$ are recruited to the cell membrane, the synthesis of PtdIns(3,4,5) P3 increases after stimulating PI3K activity by EGF $(14,15)$. However, the synthesis of PtdIns $(3,4,5) \mathrm{P} 3$ is decreased whenever Phosphatase and tensin homolog (PTEN)is recruited to the cell membrane because PTEN dephosphorylates PtdIns(3,4,5) P3to PtdIns(4,5) P2 (18). Third, the overexpression of $\mathrm{PH}$ domain might affect the specificity and affinity of binding between inositides and kinases (14). The excess amount of PH-GFP either interacts with other types of inositides or remains in the cytosol and thus affects the cellular metabolism of the cell (14).

It can be concluded that PLC $\delta 1$ - PH is likely to be entirely recruited by $\operatorname{PtdIns}(4,5) \mathrm{P} 2$ in the cell membrane. In transfected cells that expressed only a small amount of Btk-PH is likely to be recruited by $\operatorname{PtdIns}(3,4,5) \mathrm{P} 3$, while in the transfected cells that expressed more Btk$\mathrm{PH}$, it is likely to saturate the available amount of PtdIns(3,4,5)P3 pools in the cell membrane and the rest either binds with other types of 
inositides or remains in the cytoplasm. It has been shown that PH-domains are more suitable, reliable and accurate than antibodies for visualizing and studying the molecular dynamics of phospholipids in the live cells $(14,19)$. Indeed, our results showed that PH domains have more specificity and affinity than antibodies. However, there are some sideeffects, the overexpression of the $\mathrm{PH}$ domain fused with Green Fluorescent Protein (GFP) sometimes affects the physiological nature of the cell. It also can affect the binding of PHdomain with inositols. The overexpression of $\mathrm{PH}$ domain might block other kinases or proteins by interfering or interacting with their inositols which were assigned to interact with these proteins, this could affect their downstream signalling $(12,17)$. Even though $\mathrm{PH}$ domain is the best option to visualize PtdIns(4,5)P2, not all of the lipids could be visualized using these methods. For instance, PtdIns(4,5)P2 and PtdIns(3,4,5)P3 might bind with other proteins, thus rendering them incapable of binding with the $\mathrm{PH}$ probes or antibodies, which could lead to inaccurate detection (20).

It is necessary to use $\mathrm{PH}$ domain in both the fixed and the live cells as both approaches have advantages and disadvantages. For example, in the fixed cell, it can be used with high power lasers and kept in a convenient location. However, the materials that are used for the fixation and permeabilization may affect the physiological and morphological nature of the cell might also affect the dynamic nature of $\mathrm{PH}$ domain-proteins interaction. Therefore, to get more accurate results, both the fixed and the live cells should be used (14). Confocal microscopy is the most commonly used method to detect the interaction between $\mathrm{PH}$-domain and PtdIns(3,4,5)P3 and PtdIns(4,5)P2. Although confocal microscopy provides a very high quality image and has high resolution to monitor intracellular molecules, there are some limitations, such as inability to visualize the fine details of PtdIns(3,4,5) P3 and PtdIns(4,5)P2 due to diffraction limit (8). These resolution limitations are also known as the diffraction barrier, which limits the ability of optical resolution to visulize or distinguish between the smallest details of around $200 \mathrm{~nm}$ by a lateral distance around half of the wavelength of light to image the specimen (21).

Recent studies have investigated PtdIns(3,4,5) P3 and PtdIns(4,5) P2 in more details due to the availability of modern techniques that can surpass the diffraction limit, which is around $200 \mathrm{~nm}(15,22)$. For example, the single-molecule super resolution microscopies have been designed to overcome these problems and measure the spatial colocalization more precisely $(21,23)$. These methods have very high sensitivity to visualize the fine details of PtdIns(3,4,5) P3 and PtdIns(4,5) P2, and can even reach $10 \mathrm{~nm}$ in resolution. However, these methods have limitations, such as Stochastic Optical Reconstruction Microscopy (STORM) can only be used in the fixed cells and needs very efficient antibodies to detect $\operatorname{PtdIns}(3,4,5)$ P3 and PtdIns(4,5) P2 clearly $(14,19,22,23)$. Due to difficulties in direct visualization of PtdIns(3,4,5) P3 and PtdIns(4,5) P2 at cell surface by antibodies, $\mathrm{PH}$ domains were used as the effective tools that bind selectively to PtdIns(3,4,5) P3 and PtdIns(4,5) P2 (24-26). In a nutshell, our results suggest that PLC $\delta 1-\mathrm{PH}$ and Btk-PH are more suitable, reliable and accurate than antibodies for visualizing and studying the molecular dynamics of phospholipids in the live and the fixed cells.

\section{Acknowledgements}

We thank our Iraqi Ministry of Higher Education and Scientific Research and all staffs and technicians at the University of Reading, UK to their valuable support throughout the research project. The authors declare no potential conflicts of interest related to the present research and publication. 


\section{References}

1. Mashaghi S, Jadidi T, Koenderink G, Mashaghi A. Lipid Nanotechnology. Int J Mol Sci. 2013;14(2):4242-4282.

2. Wang X, Hills LB, Huang YH. Lipid and Protein Co-Regulation of PI3K Effectors Akt and Itk in Lymphocytes. Front Immunol. 2015;6:117. 3. Lemmon MA. Pleckstrin Homology $(\mathrm{PH})$ domains and phosphoinositides. Biochem Soc Symp. 2007(74):81-93.

4. Alfahad D, Alharethi S, Alharbi B, Mawlood K, Dash P. PtdIns(4,5)P2 and PtdIns(3,4,5)P3 dynamics during focal adhesions assembly and disassembly in a cancer cell line. Turk J Biol. 2020;44(6):381-92.

5. Santos CR, Schulze A. Lipid metabolism in cancer. The FEBS journal. 2012;279(15):2610-23. 6. Alharbi BF, Al-Fahad D, Dash PR. Roles of Endocytic Processes and Early Endosomes on Focal Adhesion Dynamics in MDA-MB-231 Cells 31 Cells. Rep Biochem Mol Biol. 2021;10(2):145-155.

7. Al-Fahad D, Alharbi BF, Bih CI, Dash PR. Nitric oxide may regulate focal adhesion tu rnover and cell migration in MDA-MB-231 breast cancer cells by modulating early endosome trafficking. Med J Cell Biol. 2021;9(2):60-72.

8. Balla T. Phosphoinositides: Tiny Lipids With Giant Impact on Cell Regulation. Physiol Rev. 2013;93(3):1019-1137.

9. Huang YE, Iijima M, Parent CA, Funamoto S, Firtel RA, Devreotes P. Receptor-mediated regulation of PI3Ks confines $\mathrm{PI}(3,4,5) \mathrm{P} 3$ to the leading edge of chemotaxing cells. Mol Biol Cell. 2003;14(5):1913-1922.

10. Dormann D, Weijer G, Parent CA, Devreotes PN, Weijer CJ. Visualizing PI3 kinase-mediated cell-cell signaling during Dictyostelium development. Curr Biol. 2002;12(14):1178-88.

11. Servant G, Weiner OD, Herzmark P, Balla T, Sedat JW, Bourne HR. Polarization of chemoattractant receptor signaling during neutrophil chemotaxis. Science. 2000;287(5455):1037-40.

12. Manna D, Albanese A, Park WS, Cho W. Mechanistic basis of differential cellular responses of phosphatidylinositol 3,4bisphosphate- and phosphatidylinositol 3,4,5- trisphosphate-binding pleckstrin homology domains. J Biol Chem. 2007;282(44):32093-105. 13. Varnai $P$, Rother KI, Balla T. Phosphatidylinositol 3-kinase-dependent membrane association of the Bruton's tyrosine kinase pleckstrin homology domain visualized in single living cells. J Biol Chem. 1999;274(16):10983-9.

14. Balla T, Varnai P. Visualization of cellular phosphoinositide pools with GFP-fused proteindomains. Current protocols in cell biology / editorial board, Juan S. Bonifacino... [et al.]. 2009; Chapter 24:Unit 24.4.

15. Ji C, Zhang Y, Xu P, Xu T, Lou X. Nanoscale Landscape of Phosphoinositides Revealed by Specific Pleckstrin Homology (PH) Domains Using Single-molecule Superresolution Imaging in the Plasma Membrane. J Biol Chem. 2015;290(45):26978-26993.

16. Falkenburger BH, Jensen JB, Hille B. Kinetics of $\mathrm{M}(1)$ muscarinic receptor and $G$ protein signaling to phospholipase $\mathrm{C}$ in living cells. J Gen Physiol. 2010;135(2):81-97.

17. Thapa N, Choi S, Tan X, Wise T, Anderson RA. Phosphatidylinositol Phosphate 5-Kinase Igamma and Phosphoinositide 3-Kinase/Akt Signaling Couple to Promote Oncogenic Growth. J Biol Chem. 2015;290(30):18843-54.

18. Nguyen H-N, Yang J-M, Afkari Y, Park BH, Sesaki H, Devreotes PN, et al. Engineering ePTEN, an enhanced PTEN with increased tumor suppressor activities. PNAS. 2014;111(26):E2684-E2693.

19. Idevall-Hagren O, De Camilli P. Detection and manipulation of phosphoinositides. Biochim Biophys Acta. 2015;1851(6):736-45.

20. Ji C, Lou X. Single-molecule Superresolution Imaging of Phosphatidylinositol 4,5bisphosphate in the Plasma Membrane with Novel Fluorescent Probes. J Vis Exp. 2016;(116):54466.

21. Patterson GH. Fluorescence microscopy below the diffraction limit. Semin Cell Dev Biol. 2009;20(8):886-893.

22. Wang J, Richards DA. Segregation of PIP2 and PIP3 into distinct nanoscale regions within the plasma membrane. Biol Open. 2012;1(9):857-62.

23. Warren SC, Margineanu A, Katan M, Dunsby C, French PMW. Homo-FRET Based Biosensors 
and Their Application to Multiplexed Imaging of Signalling Events in Live Cells. Int J Mol Sci. 2015;16(7):14695-716.

24. Czech MP. PIP2 and PIP3: complex roles at the cell surface. Cell. 2000;100(6):603-6.

25. Al-Fahad, D. Regulation of Focal Adhesions by PtdIns(4,5)P2 and PtdIns(3,4,5)P3 in Cancer
Cell Migration. $\mathrm{PhD}$ thesis, University of Reading.2018.

26. Al-Fahad, D. The possible role of $\operatorname{PtdIns}(4,5)$ $\mathrm{P} 2$ and PtdIns(3,4,5) P3 at the leading and trailing edges of the breast cancer cell line. I Iberoam $\mathbf{J}$ Med. 2021;3 (1): 26-32. 